\title{
Large-scale Machine Learning over Graphs
}

\author{
Yiming Yang \\ Language Technologies Institute, Carnegie Mellon University \\ 5000 Forbes Avenue, Pittsburgh, PA 15213 \\ yiming@cs.cmu.edu, http://www.cs.cmu.edu/ yiming/
}

\begin{abstract}
Graphs provide powerful representations for statistical modeling of interrelated variables (observed or latent) in a broad range of machine learning applications. Examples include learning and inference based on the dependency structures among words, documents, topics, users, items, web sites, and more. How to best leverage such dependency structures from multiple graphs with massive and heterogeneous types of nodes and relations has posed grand challenges to machine learning theory and algorithms. This talk presents our recent work in this direction focusing on three significant tasks, including 1) a novel framework for fusing multiple heterogeneous graphs into a unified product graph to enable semi-supervised multi-relational learning, 2) the first algorithmic solution for imposing analogical structures in graph-based entity/relation embedding, and 3) a new formulation of neural architecture search as a graph topology optimization problem, with simple yet powerful algorithms that automatically discover high-performing convolutional neural architectures on image recognition benchmarks, and reduce the computational cost over state-of-the-art non-differentiable techniques by several orders of magnitude.
\end{abstract}

\section{CCS Concepts/ACM Classifiers}

- General and reference

- Information systems

- Mathematics of computing

\section{Author Keywords}

Learning over graphs, multi-relational learning, analogical structure learning, neural network architecture search, large-scale optimization algorithms

\section{BIOGRAPHY}

Yiming Yang is a professor at Carnegie Mellon University with a joint appointment in the Language Technologies Institute and the Machine Learning Department. She received her Ph.D. in Computer Science from Kyoto
University (Japan), and has been a faculty member at CMU since 1996. Her research has centered on machine learning techniques and scalable algorithms and a broad range applications, including extreme-scale text categorization, semi-supervised learning over heterogeneous graphs, analogical structured learning via entity/relation embedding, time series forecasting and anomaly detection, cross-language and cross-domain transfer learning over news media data, deep neural learning for spatiotemporal modeling of social and epidemiological networks, clustering with semi-supervised metric learning for new concept discovery, large-scale optimization for online advertising, and more.

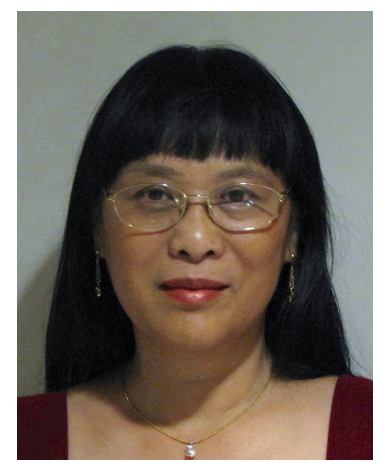

\section{REFERENCES}

[1] Hanxiao Liu, Yiming Yang: Cross-Graph Learning of Multi-Relational Associations. ICML 2016: 2235-2243

[2] Hanxiao Liu, Yuexin Wu, Yiming Yang: Analogical Inference for Multi-relational Embeddings. ICML 2017: 2168-2178

[3] Hanxiao Liu, Karen Simonyan, Yiming Yang: DARTS: Differentiable Architecture Search. arXiv:1806.09055.

[4] Hanxiao Liu, Yiming Yang: Semi-Supervised Learning with Adaptive Spectral Transform. AISTATS 2016

[5] Yiming Yang, Hanxiao Liu, Jaime G. Carbonell, Wanli Ma: Concept Graph Learning from Educational Data. WSDM 2015 